\title{
A Novel Analytical Inverse Kinematics Method for SSRMS-Type Space Manipulators Based on the POE Formula and the Paden- Kahan Subproblem
}

\author{
Yecong Wang $₫,{ }^{1}$ Xilun Ding, ${ }^{1}$ Zixin Tang, ${ }^{2}$ Chengwei Hu, ${ }^{2}$ Qingqing Wei, ${ }^{2}$ and Kun $\mathrm{Xu} \rrbracket^{1}$ \\ ${ }^{1}$ Robotics Institute, Beihang University, Beijing 100083, China \\ ${ }^{2}$ Beijing Institute of Spacecraft System Engineering, Beijing 100094, China \\ Correspondence should be addressed to Kun Xu; xk007@buaa.edu.cn
}

Received 18 November 2020; Revised 23 March 2021; Accepted 8 April 2021; Published 29 April 2021

Academic Editor: Paolo Gasbarri

Copyright ( 2021 Yecong Wang et al. This is an open access article distributed under the Creative Commons Attribution License, which permits unrestricted use, distribution, and reproduction in any medium, provided the original work is properly cited.

\begin{abstract}
Space manipulators which have a similar symmetrical structure with seven revolute joints, such as the space station remote manipulator system (SSRMS), can be called SSRMS-type space manipulators. The analytical inverse kinematics of an SSRMStype manipulator can be solved by locking a single joint; the locked joint (joint 1, 2, 6, or 7) can be determined by configuration analysis. Although widely used in establishing the kinematics of SSRMS-type manipulators, the Denavit-Hartenberg (DH) method has a singular problem when two adjacent joint axes are nearly parallel. To avoid this problem, this paper proposes a novel analytical inverse kinematics method for SSRMS-type manipulators based on the product of exponentials (POE) formula and the Paden-Kahan subproblem. Because of the symmetrical structure, an SSRMS-type manipulator degrades to two kinds of 6-degree-of-freedom (DOF) manipulators when locking a single joint (joint 1, 2, 6, or 7). The analytical inverse kinematics of these two kinds of 6-DOF manipulators is solved by combining the Paden-Kahan subproblems and geometric and algebraic methods, respectively. The proposed approach is not only singularity free compared with the traditional DH-based methods but also more accurate than the POE-based numerical solution. The simulation results verify the efficiency of the proposed approach.
\end{abstract}

\section{Introduction}

Space manipulators, such as the space station remote manipulator system (SSRMS) and the special purpose dexterous manipulator (SPDM), have played vital roles in on-orbit servicing (OOS) missions $[1,2]$ such as assembly, capture [3, 4], refueling, repair [5], and debris removal [6,7]. Compared with nonredundant space manipulators, 7-degree-of-freedom (DOF) space manipulators have advantages in obstacle avoidance, joint limit avoidance, operability enhancement [8], joint torque optimization, singularity avoidance, and joint failure $[9,10]$. There are various kinds of possible configurations for 7-DOF space manipulators. Among these configurations, the spherical-revolute-spherical (SRS) type may be the optimal version [11]. The positioning and orienting problems of an SRS-type arm can be addressed, respectively, and the analytical inverse kinematics can be easily solved subsequently [12]. However, for transportation, storage, and increasing workspace purposes, the joints of 7DOF space manipulators are always designed with broad joint movement ranges. To avoid the structural interference problem, many 7-DOF space manipulators are designed with joint offsets. At the same time, their positioning and orienting problems cannot be, respectively, resolved, and the analytical inverse kinematics cannot be easily addressed.

The SSRMS type is a typical configuration with joint offsets. The SSRMS and the Chinese space station remote manipulator system (CSSRMS), including the core module manipulator (CMM) and the experimental module manipulator [13], are typical SSRMS-type manipulators. The SPDM and the European robotic arm (ERA) have similar structures with joint offsets of different sizes. In general, an SSRMS-type space manipulator has a symmetrical design, and its joints are arranged as follows: a 3-DOF shoulder (roll-yaw-pitch), 
an elbow joint (pitch), and a 3-DOF wrist (pitch-yaw-roll), where the three axes of the shoulder/wrist do not intersect at a common point.

It is well known that the inverse kinematics of 7-DOF serial manipulators can be divided into two categories: numerical solution and analytical solution [14]. The numerical solution is usually achieved by calculating the Jacobian matrix, such as the extended Jacobian method [15], the gradient projection method [16, 17], and the weight least-norm method [18]. These methods are suitable for manipulators with different configurations as long as the Jacobian matrix equations have solutions. However, they not only are computationally expensive but also have accumulated errors. The analytical solution can be calculated by locking a single joint [19] or fixing the arm angle [12], it can directly solve all possible joint angles without knowing the initial configuration, and it is faster and more accurate than the numerical solution. Its disadvantage is that it is heavily dependent on the configurations of the manipulators. In comparison, the analytical solution is more desirable when solving the inverse kinematics of a specific 7-DOF manipulator.

For the analytical inverse kinematics of SSRMS-type manipulators, scholars have carried out related research. Based on configuration analysis, $\mathrm{Xu}$ et al. $[9,20]$ proposed a novel approach to solve the inverse kinematics of an SSRMS-type manipulator where analytical and semianalytical solutions were calculated by locking a single joint. $\mathrm{Yu}$ et al. [21] proposed a method to solve the analytical inverse kinematics of a 7-DOF space manipulator with an offset wrist. Based on constructing a virtual manipulator with a spherical wrist, the analytical inverse kinematics of a 7DOF manipulator was solved by establishing the relationship between the virtual manipulator and the actual manipulator. According to the above studies, it can be found that the $\mathrm{DH}$ method is employed to establish the kinematics of SSRMStype manipulators most of the time. Although the DH method is quite popular, it has many disadvantages. For instance, the DH method has a singular problem when the axes of two adjacent joints are nearly parallel [22]; this will cause discontinuity when it is employed in kinematics calibration of an SSRMS-type manipulator. Besides, the joint coordinates cannot be arbitrarily arranged when the $\mathrm{DH}$ method is employed. To overcome the singular problem, scholars had proposed many new methods, for instance, the $\mathrm{MDH}$ method [22], the S method [23], and the complete and parametrically continuous (CPC) method $[24,25]$, but these methods have not been widely used in modeling the kinematics of the SSRMS-type manipulators.

Another idea to establish the kinematics of a manipulator is to employ the product of exponentials (POE) formula. Due to the smooth mapping between Lie Algebras se(3) and Lie Groups SE(3), the POE formula is singularity free and has inherent advantages in modeling the kinematics of SSRMStype manipulators [26-28]; for instance, it is needless to establish the joint coordinate systems compared with the DH method. Besides, it has been widely used in the kinematics calibration of industrial manipulators [29-31]. Zhao et al. [32] discussed the singular problem of the DH and POE

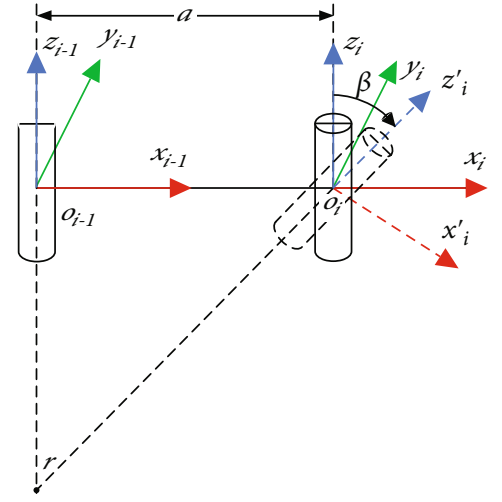

Figure 1: Singularity of the DH method.

methods in combination with a 6-DOF industrial robot with adjacent parallel joints. In our previous work, we carried out the on-orbit kinematics calibration of an SSRMS-type manipulator based on the POE formula and achieved considerable results [33]. Owing to these advantages, it is essential to establish a POE-based analytical inverse kinematics method for SSRMS-type manipulators.

In this paper, to avoid the singular problem of the $\mathrm{DH}$ method, we propose a POE-based analytical inverse kinematics method for SSRMS-type manipulators. Our approach not only avoids the singularity of the DH method but is also more accurate than the POE-based numerical solution. The remainder of this paper is organized as follows: in Section 2, the kinematics model of a typical SSRMS-type manipulator is established based on the POE formula. To determine the locked joint (joint 1, 2,6, or 7), the joints are classified into three categories by configuration analysis. Due to the symmetrical structure, the SSRMS-type manipulator degrades to two kinds of 6-DOF manipulators when locking a single joint (joint 1, 2, 6, or 7). In Section 3, we reconstruct the kinematics models of these two kinds of new 6-DOF manipulators. Analytical inverse kinematics of these two new 6-DOF manipulators is solved by combining the Paden-Kahan subproblems, geometric methods, and algebraic methods. Simulations are carried out to verify the efficiency of the proposed method in Section 4. Section 5 presents the conclusions of this paper.

\section{The Kinematics and Model Analysis}

2.1. Singularity Analysis of the DH Method. When establishing the kinematics of a manipulator, the DH method is the most popular, and it has four parameters: twist angle $\alpha_{i-1}$, link length $a_{i-1}$, joint angle $\theta_{i}$, and translation distance $d_{i}$, to represent the relationship between two adjacent joints. However, when the adjacent joint axes of a manipulator are parallel or nearly parallel, the corresponding $\mathrm{DH}$ parameters are discontinuous, which leads to the singularity of the $\mathrm{DH}$ method. Related studies have demonstrated the singularity of the DH method [22-25]. For instance, as shown in Figure 1, the nominal DH parameters of the $i$ th link are $\alpha_{i-1}=0^{\circ}, a_{i-1}=a, \theta_{i}=0^{\circ}$, and $d_{i}=0$. If the $i$ th 


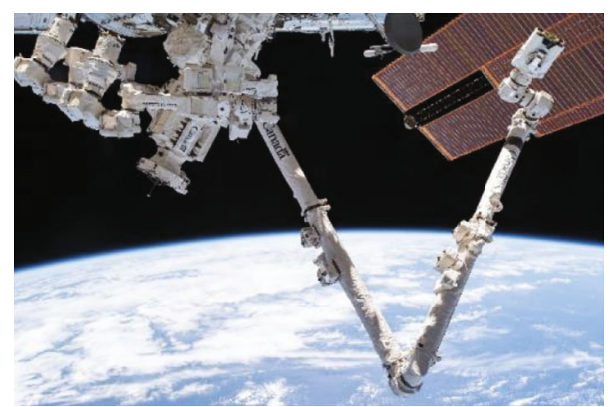

(a) SSRMS (courtesy NASA)

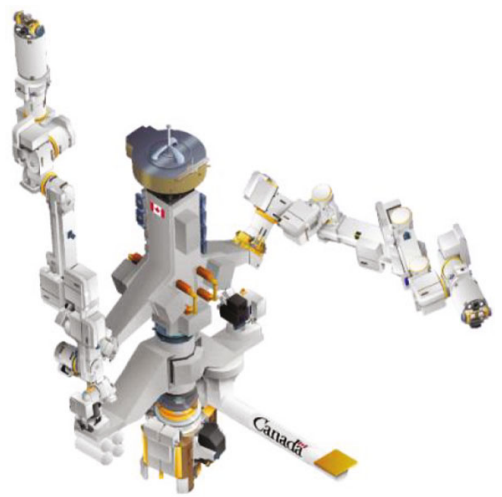

(c) SPDM (courtesy NASA)

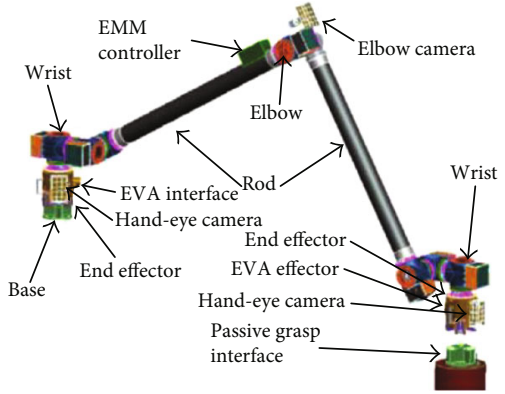

(b) EMM of CSSRMS [13]

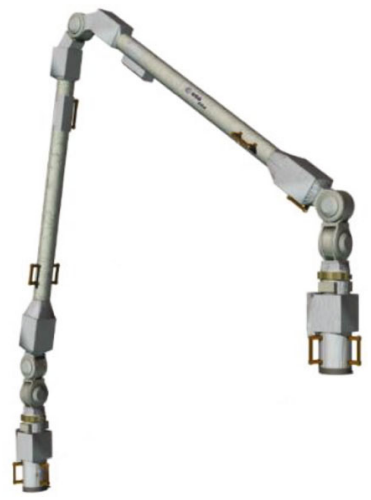

(d) ERA (courtesy ESA)

FIgURE 2: Typical SSRMS-type space manipulators.

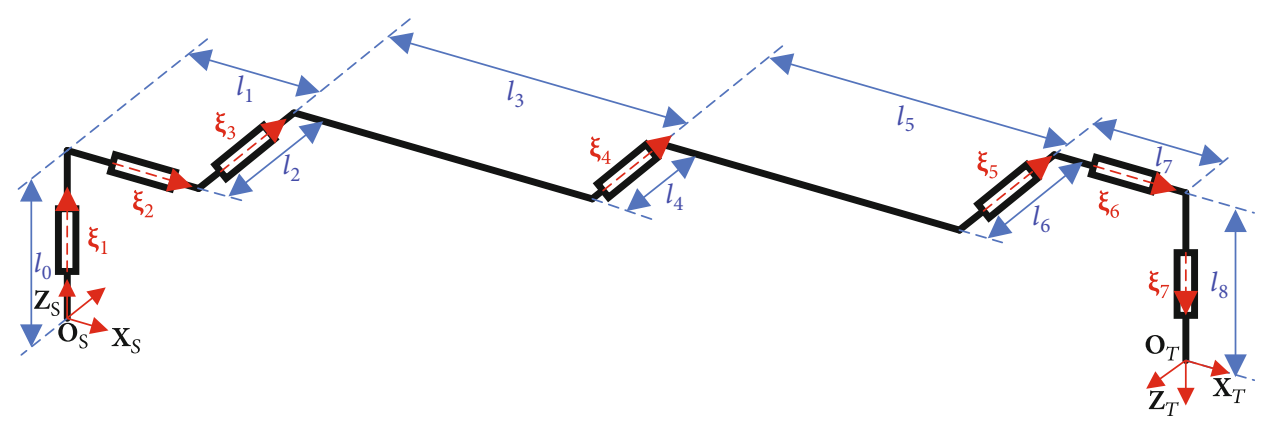

FIGURE 3: The kinematics model of an SSRMS-type manipulator.

joint coordinate system rotates a small angle $\beta(\beta \longrightarrow 0)$ about axis $y_{i}$, axes $z_{i-1}$ and $z_{i}$ will intersect at a common point $r$; the corresponding $\mathrm{DH}$ parameters will change from nominal values to actual values: $\alpha_{i-1}=0^{\circ}, a_{i-1}=0$, $\theta_{i}=0^{\circ}$, and $d_{i}=0$. It can be found that when the adjacent joint axes are parallel, small deviations of the adjacent parallel joint axes lead to huge changes in the $\mathrm{DH}$ parameters. In fact, due to the manufacturing and assembly errors, there is no guarantee that the adjacent joint axes are completely parallel. Therefore, due to the singularity of the DH method, it is not suitable for establishing the kinematics of manipulators with adjacent parallel joints, especially SSRMS-type space manipulators with three adjacent parallel joints and large structural size.
2.2. The Kinematics Model Based on the POE Formula. SSRMS-type space manipulators, such as the SSRMS, CSSRMS, SPDM, and ERA, play key roles in various OOS missions. As shown in Figure 2, they all have seven revolute joints and a similar joint arrangement: shoulder (roll-yaw-pitch), elbow (pitch), and wrist (pitch-yaw-roll). Although they differ in structural sizes and joint offset, the configuration of SSRMS-type manipulators can be expressed in a unified form, as shown in Figure 3.

Due to the smooth mapping between Lie Algebras se(3) and Lie Groups SE(3), the POE formula is employed to establish the kinematics of a typical SSRMS-type manipulator. As shown in Figure 3, we attach a base coordinate system $\{S\}$ to the base link and a tool coordinate system $\{T\}$ to the end 
TABLE 1: The kinematics parameters of an SSRMS-type manipulator.

\begin{tabular}{|c|c|c|c|c|c|c|c|c|c|c|c|}
\hline $\begin{array}{l}\text { No. } \\
i\end{array}$ & Axis & $\begin{array}{l}\text { direction } \\
\boldsymbol{\omega}_{i} \\
\end{array}$ & Position $\mathbf{r}_{i}$ & \multirow[b]{4}{*}{$\mathbf{g}_{S T}(\mathbf{0})=$} & \multicolumn{6}{|c|}{ The initial pose of the end effector } & \\
\hline 1 & {$[0$} & $\left.\begin{array}{ll}0 & 1\end{array}\right]^{T}$ & {$\left[\begin{array}{lll}0 & 0 & 0\end{array}\right]^{T}$} & & & & & & & & \\
\hline 2 & {$[1$} & $\left.\begin{array}{ll}0 & 0\end{array}\right]^{T}$ & {$\left[\begin{array}{lll}0 & 0 & l_{0}\end{array}\right]^{T}$} & & {$[\mathbf{R}(\mathbf{0})$} & $\mathbf{p}(\mathbf{0})]$ & & & & & \\
\hline 3 & {$[0$} & $\left.\begin{array}{ll}1 & 0\end{array}\right]^{T}$ & {$\left[\begin{array}{lll}l_{1} & 0 & l_{0}\end{array}\right]^{T}$} & & 0 & 1 & , where $\mathbf{R}(\mathbf{0})=$ & 0 & -1 & 0 & and $\mathbf{p}(\mathbf{0})=$ \\
\hline 4 & {$[0$} & $1 \quad 0]^{T}$ & {$\left[\begin{array}{lll}l_{1}+l_{3} & 0 & l_{0}\end{array}\right]^{T}$} & & & & {$\left[l_{1}+l_{3}+l_{5}+l_{7}\right.$} & & 0 & -1 & \\
\hline 5 & {$[0$} & $\left.\begin{array}{ll}1 & 0\end{array}\right]^{T}$ & {$\left[l_{1}+l_{3}+l_{5} \quad 0 \quad l_{0}\right]^{T}$} & & & & $l_{2}+l_{4}+l_{6}$ & & & & \\
\hline 6 & {$[1$} & $\left.\begin{array}{ll}0 & 0\end{array}\right]^{T}$ & {$\left[\begin{array}{lll}0 & l_{2}+l_{4}+l_{6} & l_{0}\end{array}\right]^{T}$} & & & & 0 & & & & \\
\hline 7 & {$[0$} & $0 \quad-1]^{T}$ & {$\left[\begin{array}{lll}l_{1}+l_{3}+l_{5}+l_{7} & l_{2}+l_{4}+l_{6} & 0\end{array}\right]^{T}$} & & & & & & & & \\
\hline
\end{tabular}

effector. The pose of the tool frame $\{T\}$ with respect to the base frame $\{S\}$ can be expressed with a homogeneous transformation matrix g. All these matrixes form a rigid motion group, and an element of this group can be expressed as $\mathbf{g} \in \mathrm{SE}(3)$

$$
\mathbf{g}=\left[\begin{array}{ll}
\mathbf{R} & \mathbf{p} \\
\mathbf{0} & 1
\end{array}\right]
$$

where the $3 \times 3$ matrix $\mathbf{R} \in \mathrm{SO}(3)$ is a special orthogonal matrix describing the coordinate axes of the tool frame $\{T\}$ in the base frame $\{S\}$, and the $3 \times 1$ vector $\mathbf{p} \in \mathbb{R}^{3}$ is the position vector of the origin of the tool frame $\{T\}$ in the base frame $\{S\}$.

Besides, each joint is associated with a twist $\xi_{i}(i=1$, $2, \cdots, 7)$, the twist coordinate of the $i$ th joint $\xi_{i}$ in the base frame $\{S\}$ :

$$
\boldsymbol{\xi}_{i}=\left[\begin{array}{c}
\mathbf{v}_{i} \\
\boldsymbol{\omega}_{i}
\end{array}\right]=\left[\begin{array}{c}
-\boldsymbol{\omega}_{i} \times \mathbf{r}_{i} \\
\boldsymbol{\omega}_{i}
\end{array}\right]
$$

where $\boldsymbol{\omega}_{i}$ is the unit vector along the $i$ th joint axis and $\mathbf{r}_{i}$ is the position vector located at any point of the $i$ th joint axis. Therefore, the twist coordinate of the $i$ th revolute joint $\xi_{i}$ in the base frame $\{S\}$ satisfies the following constraints:

$$
\left\{\begin{array}{l}
\left\|\boldsymbol{\omega}_{i}\right\|=1 \\
\boldsymbol{\omega}_{i}^{T} \mathbf{v}_{i}=0
\end{array}\right.
$$

where $\|\cdot\|$ represents the 2-norm of a vector.

The twist coordinate of the $i$ th joint axis $\xi_{i}$ can also be expressed in a matrix form $\widehat{\xi}_{i} \in \operatorname{se}(3)$ :

$$
\widehat{\boldsymbol{\xi}}_{i}=\left[\begin{array}{cc}
\widehat{\boldsymbol{\omega}}_{i} & \mathbf{v}_{i} \\
\mathbf{0} & 0
\end{array}\right]
$$

where the symbol $\wedge$ represents a map from a 6-dimensional (or 3-dimensional) vector space to Lie Algebra space se(3)

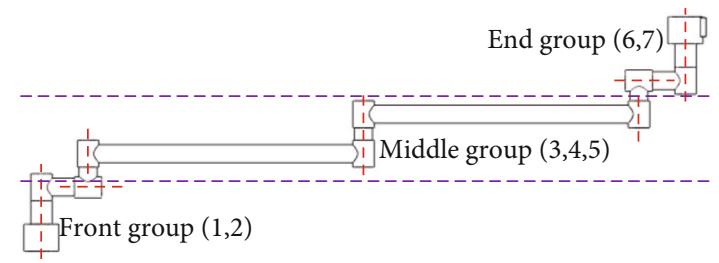

FIgURe 4: Classification of the joints.

$(\operatorname{so}(3))$ and $\widehat{\boldsymbol{\omega}}_{i}$ is the antisymmetric matrix of $\boldsymbol{\omega}_{i}$ and can be expressed as

$$
\widehat{\boldsymbol{\omega}}_{i}=\left[\begin{array}{ccc}
0 & -\omega_{z} & \omega_{y} \\
\omega_{z} & 0 & -\omega_{x} \\
-\omega_{y} & \omega_{x} & 0
\end{array}\right] .
$$

The forward kinematics of the SSRMS-type manipulator can be given by using the POE formula:

$$
\mathbf{g}_{S T}(\boldsymbol{\theta})=e^{\xi \wedge_{1} \theta_{1}} e^{\xi \wedge_{2} \theta_{2}} e^{\xi \wedge_{3} \theta_{3}} e^{\xi \wedge_{4} \theta_{4}} e^{\xi \wedge_{5} \theta_{5}} e^{\xi \wedge_{6} \theta_{6}} e^{\xi \wedge_{7} \theta_{7}} \mathbf{g}_{S T}(\mathbf{0})
$$

where $\mathbf{g}_{S T}(\boldsymbol{\theta})$ and $\mathbf{g}_{S T}(\mathbf{0})$ represent the actual and initial pose of the tool frame $\{T\}$ in the base frame $\{S\}$, respectively, and $\theta_{i}, i=1,2, \cdots, 7$, represents the position of the $i$ th joint. $e^{\xi \wedge_{i} \theta_{i}}$ can be expressed as

$e^{\xi \wedge_{i} \theta_{i}}=\left[\begin{array}{cc}e^{\omega \wedge_{i} \theta_{i}} & \left(\mathbf{I}-e^{\boldsymbol{\omega} \wedge_{i} \theta_{i}}\right)\left(\boldsymbol{\omega}_{i} \times \mathbf{v}_{i}\right)+\boldsymbol{\omega}_{i} \boldsymbol{\omega}_{i}^{T} \mathbf{v}_{i} \theta \\ \mathbf{0} & 1\end{array}\right], \quad \boldsymbol{\omega}_{i} \neq 0$,

$e^{\boldsymbol{\omega} \wedge_{i} \theta_{i}}=\mathbf{I}+\widehat{\boldsymbol{\omega}}_{i} \sin \theta_{i}+\widehat{\boldsymbol{\omega}}_{i} \sin \theta_{i}+\widehat{\boldsymbol{\omega}}_{i}^{2}\left(1-\cos \theta_{i}\right)$.

The kinematics parameters of the SSRMS-type manipulator are listed in Table 1.

2.3. Configuration Analysis. After establishing the forward kinematics of the SSRMS-type manipulator by using the POE formula, the configuration analysis is required to determine which joint can be locked to solve the analytical inverse kinematics of the SSRMS-type manipulator. It is 


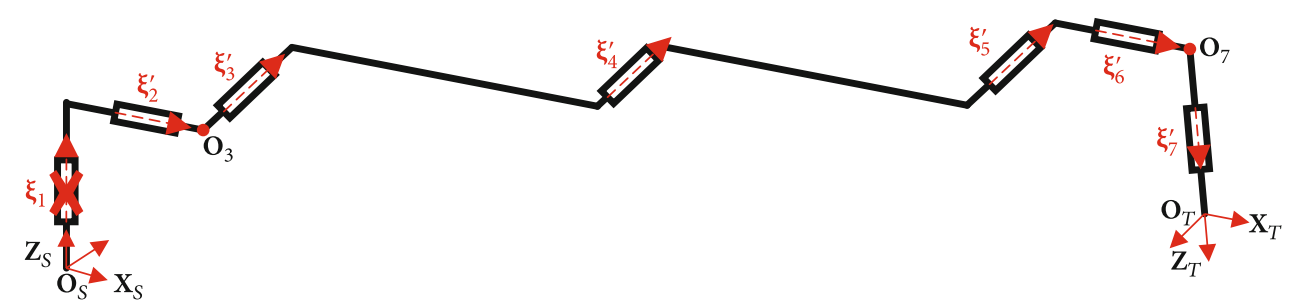

FigURE 5: The reconstructed 6-DOF manipulator for locking the 1st joint.

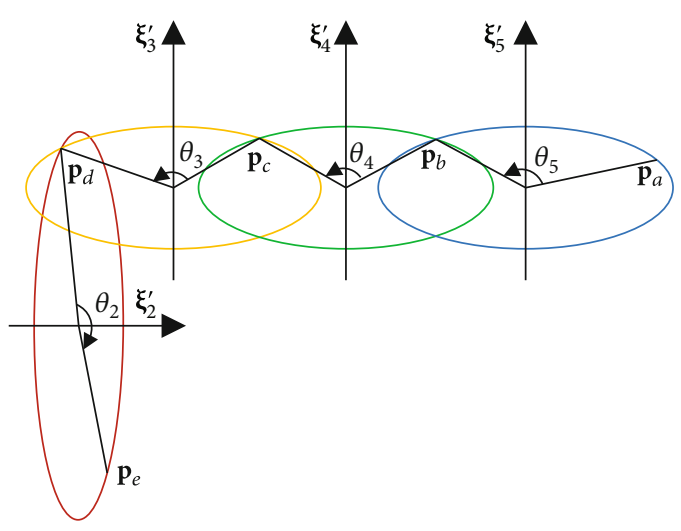

Figure 6: The screw motion of joints 2, 3, 4, and 5.

well known that there are two sufficient conditions to judge whether a 6-DOF manipulator has analytical inverse kinematics solutions:

(a) Three adjacent revolute joint axes intersect at a common point

(b) Three adjacent revolute joint axes are parallel

By analyzing the configuration of the SSRMS-type manipulators, it can be found that there are three adjacent parallel joints (joints 3, 4, and 5). If all these three adjacent parallel joints are not locked in the new 6-DOF manipulator, there exist analytical solutions. In contrast, if any one of them is locked, the analytical solution condition for the new 6DOF manipulator is not satisfied. To facilitate analysis, the joints of an SSRMS-type manipulator are classified into three groups [9]: the front group (joints 1 and 2), the middle group (joints 3, 4, and 5), and the end group (joints 6 and 7), as shown in Figure 4. Then, the analytical inverse kinematics of an SSRMS-type manipulator can be solved only when a joint in the front or end group (joint 1, 2, 6, or 7) is locked.

\section{Analytical Inverse Kinematics for Locking a Single Joint}

When locking a joint in the front or end group (joint 1, 2, 6, or 7), the SSRMS-type manipulator degrades to a new 6-DOF manipulator; the analytical inverse kinematics of the new 6DOF manipulator can be solved by using the Paden-Kahan problems. The three basic Paden-Kahan subproblems [34] are listed as follows:
(1) Rotation about a single axis

(2) Rotation about two subsequent axes

(3) Rotation to a given distance

Due to the symmetrical structure of the SSRMS-type manipulators, the cases for locking a front or end joint (joints 1 and 7 or joints 2 and 6) are similar. Hence, only the analytical inverse kinematics for locking a joint in the front group (joints 1 and 2) are detailed.

3.1. Solving $\theta_{2}$ When the First Joint Is Locked. When the first joint is locked at a fixed position $\theta_{1}^{L}$, the SSRMS-type manipulator degrades to a new 6-DOF manipulator. As shown in Figure 5, the forward kinematics of the new 6-DOF manipulator can be written in the following form:

$$
\mathbf{g}_{S T}(\boldsymbol{\theta})=e^{\xi \wedge_{2}{ }^{\prime} \theta_{2}} e^{\xi \wedge_{3}{ }^{\prime} \theta_{3}} e^{\xi \wedge_{4}^{\prime} \theta_{4}} e^{\xi \wedge_{5}{ }^{\prime} \theta_{5}} e^{\xi \wedge_{6}{ }^{\prime} \theta_{6}} e^{\xi \wedge_{7}{ }^{\prime} \theta_{7}} e^{\xi \wedge_{1} \theta_{1}^{L}} \mathbf{g}_{S T}(\mathbf{0})
$$

where $\xi_{i}^{\prime}$ represents the $i$ th joint twist coordinate of the new 6-DOF manipulator and $\xi_{i}^{\prime}=\operatorname{Ad}_{e^{\xi} \wedge_{1} \theta_{1}} \xi_{i}(i=2,3, \cdots, 7)$. $\mathrm{Ad}_{T}$ represents the adjoint transformation associated with

$$
\mathbf{T}=\left[\begin{array}{cc}
\mathbf{R}_{T} & \mathbf{p}_{T} \\
\mathbf{0} & 1
\end{array}\right],
$$

which transforms a joint twist from one coordinate system to another, and it can be written in the following form:

$$
\operatorname{Ad}_{T}=\left[\begin{array}{cc}
\mathbf{R}_{T} & \widehat{\mathbf{p}}_{T} \mathbf{R}_{T} \\
\mathbf{0} & \mathbf{R}_{T}
\end{array}\right] .
$$

Right multiplying both sides of equation (8) by $g_{S T}^{-1}(0)$ $e^{-\xi \wedge_{1} \theta_{1}^{L}}$ gives the following relationship:

$$
\begin{aligned}
\mathbf{g}_{S T}(\boldsymbol{\theta}) \mathbf{g}_{S T}^{-1}(\mathbf{0}) e^{-\xi \wedge_{1} \theta_{1}^{L}} & =e^{\xi \wedge_{2}^{\prime} \theta_{2}} e^{\xi \wedge_{3}^{\prime} \theta_{3}} e^{\xi \wedge_{4}^{\prime} \theta_{4}} e^{\xi \wedge_{5}^{\prime} \theta_{5}} e^{\xi \wedge_{6}^{\prime} \theta_{6}} e^{\xi \wedge_{7}^{\prime} \theta_{7}} \\
& =\mathbf{g}_{S T_{-} 1}(\boldsymbol{\theta}) .
\end{aligned}
$$




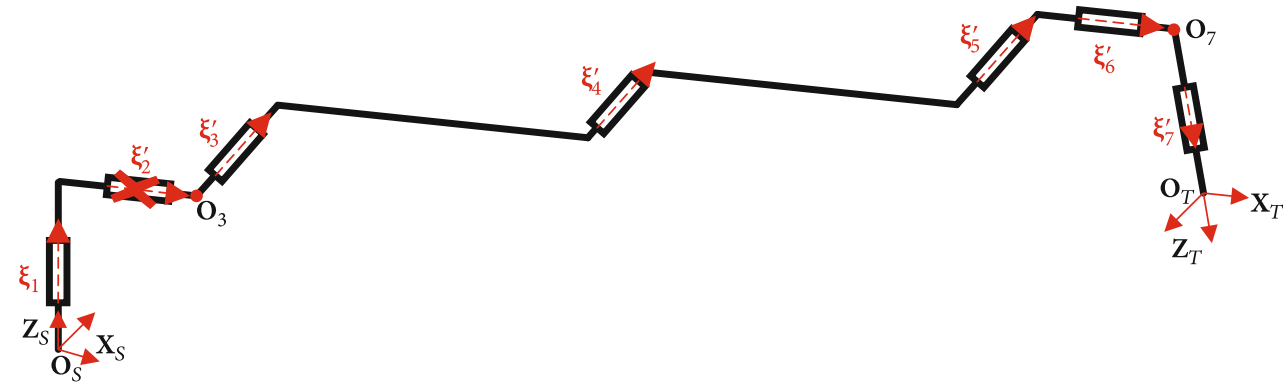

FIgURE 7: The reconstructed 6-DOF manipulator for locking the 2nd joint.

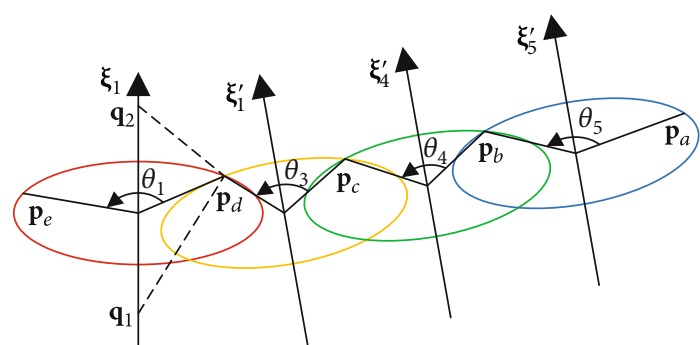

FIgURE 8: The screw motion of joints $1,3,4$, and 5 .

As shown in Figure 5, point $\mathbf{O}_{3}$ is the intersection of the second and third joint axes and point $\mathbf{O}_{7}$ is the intersection of the 6th and 7 th joint axes. Assume that $\mathbf{p}_{f 0}$ and $\mathbf{p}_{a 0}$ are the initial positions of $\mathbf{O}_{3}$ and $\mathbf{O}_{7}$ when all joints of the SSRMS-type manipulator are at zero degrees and $\mathbf{p}_{f}$ and $\mathbf{p}_{a}$ are the initial positions of points $\mathbf{O}_{3}$ and $\mathbf{O}_{7}$ when joint 1 is locked at $\theta_{1}^{L}$. Hence, $\mathbf{p}_{a}$ and $\mathbf{p}_{f}$ can be expressed as

$$
\begin{aligned}
& {\left[\begin{array}{c}
\mathbf{p}_{f} \\
1
\end{array}\right]=e^{\xi \wedge_{1} \theta_{1}^{L}}\left[\begin{array}{c}
\mathbf{p}_{f 0} \\
1
\end{array}\right],} \\
& {\left[\begin{array}{c}
\mathbf{p}_{a} \\
1
\end{array}\right]=e^{\xi \wedge_{1} \theta_{1}^{L}}\left[\begin{array}{c}
\mathbf{p}_{a 0} \\
1
\end{array}\right] .}
\end{aligned}
$$

By right multiplying both sides of equation (11) by $\mathbf{p}_{a}$, the screw motion of point $\mathbf{O}_{7}$ in joints 2, 3, 4 , and 5 can be obtained:

$$
\mathbf{g}_{S T_{-} 1}(\boldsymbol{\theta})\left[\begin{array}{c}
\mathbf{p}_{a} \\
1
\end{array}\right]=e^{\xi \wedge_{2}^{\prime} \theta_{2}} e^{\xi \wedge_{3}^{\prime} \theta_{3}} e^{\xi \wedge_{4}^{\prime} \theta_{4}} e^{\xi \wedge_{5}^{\prime} \theta_{5}}\left[\begin{array}{c}
\mathbf{p}_{a} \\
1
\end{array}\right]=\left[\begin{array}{c}
\mathbf{p}_{e} \\
1
\end{array}\right],
$$

where $\mathbf{p}_{a}$ and $\mathbf{p}_{e}$ are the initial and final positions of point $\mathbf{O}_{7}$, respectively. As shown in Figure $6, \mathbf{p}_{b}$ is obtained by rotating $\mathbf{p}_{a}$ around the 5 th joint axis by $\theta_{5}, \mathbf{p}_{c}$ is obtained by rotating $\mathbf{p}_{b}$ around the 4 th joint axis by $\theta_{4}, \mathbf{p}_{d}$ is obtained by rotating $\mathbf{p}_{c}$ around the 3 rd joint axis by $\theta_{3}$, and $\mathbf{p}_{e}$ is obtained by rotating $\mathbf{p}_{d}$ around the 2 nd joint axis by $\theta_{2}$.

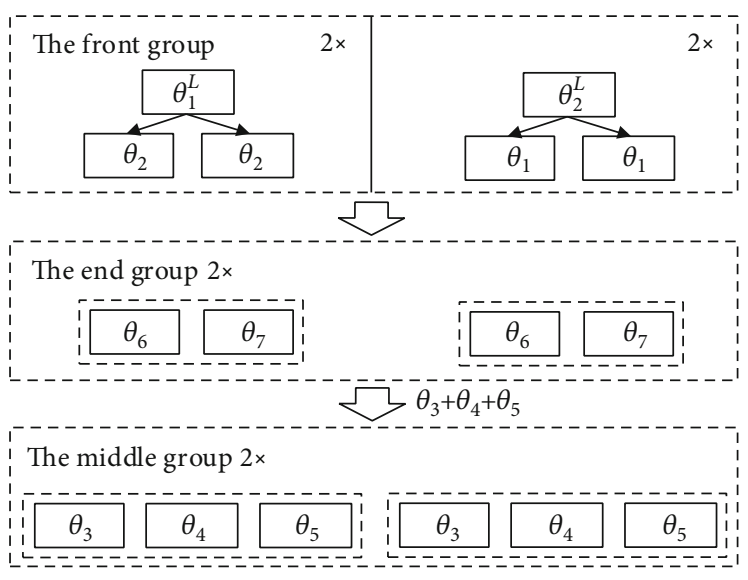

Figure 9: Flowchart of the solution process.

According to the geometrical relationship shown in Figure 6, we can obtain the following equation set:

$$
\left\{\begin{array}{l}
\left(\mathbf{p}_{d}-\mathbf{p}_{a}\right) \cdot \boldsymbol{\omega}_{3}^{\prime}=0, \\
\left(\mathbf{p}_{d}-\mathbf{p}_{f}\right) \cdot \boldsymbol{\omega}_{2}^{\prime}=0, \\
\left\|\mathbf{p}_{d}-\mathbf{p}_{f}\right\|=\left\|\mathbf{p}_{e}-\mathbf{p}_{f}\right\| .
\end{array}\right.
$$

$\mathbf{p}_{d}$ can be obtained using equation (14).

The screw motion of point $\mathbf{O}_{7}$ in the second joint can be expressed as

$$
e^{\xi \wedge_{2}^{\prime} \theta_{2}}\left[\begin{array}{c}
\mathbf{p}_{d} \\
1
\end{array}\right]=\left[\begin{array}{c}
\mathbf{p}_{e} \\
1
\end{array}\right]
$$

Hence, $\theta_{2}$ can be obtained using Paden-Kahan subproblem 1 [34].

3.2. Solving $\theta_{1}$ When the Second Joint Is Locked. When the second joint of the SSRMS-type manipulator is locked at a fixed position $\theta_{2}^{L}$, the SSRMS-type manipulator degrades to another new 6-DOF manipulator. As shown in Figure 7, 
TABLE 2: The eight sets of solutions for locking the first joint.

\begin{tabular}{lccccccc}
\hline No. $i$ & $\theta_{1}^{L}\left(^{\circ}\right)$ & $\theta_{2}\left(^{\circ}\right)$ & $\theta_{3}\left(^{\circ}\right)$ & $\theta_{4}\left(^{\circ}\right)$ & $\theta_{5}\left(^{\circ}\right)$ & $\theta_{6}\left(^{\circ}\right)$ & $\theta_{7}\left({ }^{\circ}\right)$ \\
\hline 1 & 30 & -84.6327446802879 & 135.936469380010 & -49.5026276247550 & 52.8462511448701 & 130.991940778053 & -108.457688216213 \\
2 & 30 & -84.6327446802879 & 88.5675124089909 & 49.5026276247550 & 1.20995286637878 & 130.991940778053 & -108.457688216213 \\
3 & 30 & -84.6327446802879 & 159.151456789031 & -68.0612040974591 & -131.810159791447 & -130.991940778053 & 71.5423117837873 \\
4 & 30 & -84.6327446802879 & 94.2150692495295 & 68.0612040974592 & 157.003819553136 & -130.991940778053 & 71.5423117837873 \\
5 & 30 & 60.0000000000000 & 77.8419290875575 & -49.9999999999999 & 52.1580709124424 & 30.0000000000000 & 22.5000000000000 \\
6 & 30 & 60.0000000000000 & 30.0000000000000 & 50.0000000000000 & 0.0000000000000 & 30.0000000000000 & 22.5000000000000 \\
7 & 30 & 60.0000000000000 & 100.714460683408 & -67.6513565340774 & -133.063104149330 & -30.0000000000000 & -157.500000000000 \\
8 & 30 & 60.0000000000000 & 36.1638929853303 & 67.6513565340774 & 156.184750480592 & -30.0000000000000 & -157.500000000000 \\
\hline
\end{tabular}

the forward kinematics of the new 6-DOF manipulator can be written in the following form:

$$
\mathbf{g}_{S T}(\boldsymbol{\theta})=e^{\xi \wedge_{1} \theta_{1}} e^{\xi \wedge_{3}^{\prime} \theta_{3}} e^{\xi \wedge_{4}^{\prime} \theta_{4}} e^{\xi \wedge_{5}^{\prime} \theta_{5}} e^{\xi \wedge_{6}^{\prime} \theta_{6}} e^{\xi \wedge_{7}^{\prime} \theta_{7}} e^{\xi \wedge_{2} \theta_{2}^{L}} \mathbf{g}_{S T}(\mathbf{0}),
$$

where $\xi_{i}^{\prime}$ represents the $i$ th joint twist of the new 6-DOF manipulator; $\xi_{i}^{\prime}=\operatorname{Ad}_{e^{\xi \wedge_{2} \theta_{2}^{L}}} \boldsymbol{\xi}_{i}(i=3,4, \cdots, 7)$.

As shown in Figure 7, $\mathbf{O}_{7}$ is the intersection of the 6 th and 7 th joint axes. Assume that $\mathbf{p}_{a 0}$ is the initial position of point $\mathbf{O}_{7}$ when all joints of the SSRMS-type manipulator are at zero degrees. $\mathbf{p}_{a}$ is the position of $\mathbf{O}_{7}$ when the second joint is locked at $\theta_{2}^{L}$. We have

$$
\left[\begin{array}{c}
\mathbf{p}_{a} \\
1
\end{array}\right]=e^{\xi \wedge_{2} \theta_{2}^{L}}\left[\begin{array}{c}
\mathbf{p}_{a 0} \\
1
\end{array}\right]
$$

Right multiplying both sides of equation (16) by $\mathbf{g}_{S T}^{-1}(\mathbf{0})$ $e^{-\xi \wedge_{2} \theta_{2}^{L}}$ gives the following relationship:

$$
\begin{aligned}
\mathbf{g}_{S T}(\boldsymbol{\theta}) \mathbf{g}_{S T}^{-1}(\mathbf{0}) e^{-\xi \wedge_{2} \theta_{2}^{L}} & =e^{\xi \wedge_{1} \theta_{1}} e^{\xi \wedge_{3}{ }^{\prime} \theta_{3}} e^{\xi \wedge_{4}{ }^{\prime} \theta_{4}} e^{\xi \wedge_{5}{ }^{\prime} \theta_{5}} e^{\xi \wedge_{6}{ }^{\prime} \theta_{6}} e^{\xi \wedge_{7}{ }^{\prime} \theta_{7}} \\
& =\mathbf{g}_{S T_{-} 1}(\boldsymbol{\theta}) .
\end{aligned}
$$

By right multiplying both sides of equation (11) by $\mathbf{p}_{a}$, the screw motion of point $\mathbf{O}_{7}$ in joints $1,3,4$, and 5 can be obtained:

$$
\mathbf{g}_{S T_{-} 1}(\boldsymbol{\theta})\left[\begin{array}{c}
\mathbf{p}_{a} \\
1
\end{array}\right]=e^{\xi \wedge_{1} \theta_{1}} e^{\xi \wedge_{3}^{\prime} \theta_{3}} e^{\xi \wedge_{4}^{\prime} \theta_{4}} e^{\xi \wedge_{5}^{\prime} \theta_{5}}\left[\begin{array}{c}
\mathbf{p}_{a} \\
1
\end{array}\right]=\left[\begin{array}{c}
\mathbf{p}_{e} \\
1
\end{array}\right],
$$

where $\mathbf{p}_{a}$ and $\mathbf{p}_{e}$ are the initial and final positions of point $\mathbf{O}_{7}$, respectively. As shown in Figure $8, \mathbf{p}_{b}$ is obtained by rotating $\mathbf{p}_{a}$ around the 5 th joint axis by $\theta_{5}, \mathbf{p}_{c}$ is obtained by rotating $\mathbf{p}_{b}$ around the 4 th joint axis by $\theta_{4}, \mathbf{p}_{d}$ is obtained by rotating $\mathbf{p}_{c}$ around the 3 rd joint axis by $\theta_{3}, \mathbf{p}_{e}$ is obtained by rotating $\mathbf{p}_{d}$ around the 1 st joint axis by $\theta_{1}$, and $\mathbf{q}_{1}$ and $\mathbf{q}_{2}$ are two arbitrarily different points on the first joint axis. According to the geometrical relationship shown in Figure 8, we can obtain the following equation set:

$$
\left\{\begin{array}{l}
\left\|e^{\xi \wedge_{1} \theta_{1}}\left(e^{\xi \wedge_{3}{ }^{\prime} \theta_{3}} e^{\xi \wedge_{4}{ }^{\prime} \theta_{4}} e^{\xi \wedge_{5}{ }^{\prime} \theta_{5}} \mathbf{p}_{a}-\mathbf{q}_{1}\right)\right\|=\left\|e^{\xi \wedge_{3} \theta_{3}} e^{\xi \wedge_{4}{ }^{\prime} \theta_{4}} e^{\xi \wedge_{5}{ }^{\prime} \theta_{5}} \mathbf{p}_{a}-\mathbf{q}_{1}\right\|, \\
\left\|e^{\xi \wedge_{1} \theta_{1}} e^{\xi \wedge_{3}{ }^{\prime} \theta_{3}} e^{\xi \wedge_{4}{ }^{\prime} \theta_{4}} e^{\xi \wedge_{5}{ }^{\prime} \theta_{5}} \mathbf{p}_{a}-\mathbf{q}_{1}\right\|=\left\|e^{\xi \wedge_{3}{ }^{\prime} \theta_{3}} e^{\xi \wedge_{4}{ }^{\prime} \theta_{4}} e^{\xi \wedge_{5}{ }^{\prime} \theta_{5}} \mathbf{p}_{a}-\mathbf{q}_{2}\right\|=\left\|\mathbf{p}_{e}-\mathbf{q}_{1}\right\|, \\
\left\|e^{\xi \wedge_{1} \theta_{1}} e^{\xi \wedge_{3} \theta_{3}} e^{\xi \wedge_{4}{ }^{\prime} \theta_{4}} e^{\xi \wedge_{5}{ }^{\prime} \theta_{5}} \mathbf{p}_{a}-\mathbf{q}_{2}\right\|=\left\|e^{\xi \wedge_{3}{ }^{\prime} \theta_{3}} e^{\xi \wedge_{4}{ }^{\prime} \theta_{4}} e^{\xi \wedge_{5}{ }^{\prime} \theta_{5}} \mathbf{p}_{a}-\mathbf{q}_{1}\right\|=\left\|\mathbf{p}_{e}-\mathbf{q}_{2}\right\|
\end{array}\right.
$$

According to equations (19) and (20), we can get

$$
\left\{\begin{array}{l}
\left\|\mathbf{p}_{d}-\mathbf{q}_{1}\right\|=\left\|\mathbf{p}_{e}-\mathbf{q}_{1}\right\|, \\
\left\|\mathbf{p}_{d}-\mathbf{q}_{2}\right\|=\left\|\mathbf{p}_{e}-\mathbf{q}_{2}\right\|, \\
\left(\mathbf{p}_{d}-\mathbf{p}_{a}\right) \boldsymbol{\omega}_{3}^{\prime}=0 .
\end{array}\right.
$$

$\mathbf{p}_{d}$ can be obtained by solving the above equation set. According to Figure 8, the screw motion of point $\mathbf{O}_{7}$ in the second joint can be expressed as

$$
e^{\xi \wedge_{1}^{\prime} \theta_{1}}\left[\begin{array}{c}
\mathbf{p}_{d} \\
1
\end{array}\right]=\left[\begin{array}{c}
\mathbf{p}_{e} \\
1
\end{array}\right]
$$

Hence, $\theta_{1}$ can be obtained by using Paden-Kahan subproblem 1 [34].

3.3. Solving $\theta_{3}, \theta_{4}, \theta_{5}, \theta_{6}$, and $\theta_{7}$. According to Sections 3.1 and 3.2 , both $\theta_{1}$ and $\theta_{2}$ can be solved. Left multiplying both 
TABLE 3: The eight sets of solutions for locking the second joint.

\begin{tabular}{lccccccc}
\hline $\begin{array}{l}\text { No. } \\
i\end{array}$ & $\theta_{1}\left({ }^{\circ}\right)$ & $\theta_{2}^{L}$ & $\theta_{3}\left({ }^{\circ}\right)$ & $\theta_{4}\left({ }^{\circ}\right)$ & $\theta_{5}\left({ }^{\circ}\right)$ & $\theta_{6}\left({ }^{\circ}\right)$ & $\theta_{7}\left({ }^{\circ}\right)$ \\
\hline 1 & -129.239754823904 & 45 & 162.018988544233 & -52.260284762302660 & 31.003837249502922 & 104.505649369635 & -142.495639227140 \\
2 & -129.239754823904 & 45 & 112.028927802159 & 52.260284762302774 & -23.5266715330279 & 104.505649369635 & -142.495639227140 \\
3 & -129.239754823904 & 45 & 174.819256106276 & -48.2841313736117 & -165.772583701231 & -104.505649369635 & 37.5043607728603 \\
4 & -129.239754823904 & 45 & 128.609425992290 & 48.2841313736117 & 143.868983665532 & -104.505649369635 & 37.5043607728603 \\
5 & 30.0000000000000 & 45 & 50.0000000000000 & -45.0000000000000 & 40.0000000000000 & 60.0000000000000 & 22.5000000000000 \\
6 & 30.0000000000000 & 45 & 6.91702734831439 & 45.0000000000000 & -6.91702734831438 & 60.00000000000000 & 22.5000000000000 \\
7 & 30.0000000000000 & 45 & 69.3878143138167 & -55.2214803970355 & -149.166333916781 & -60.0000000000000 & -157.500000000000 \\
8 & 30.0000000000000 & 45 & 16.5868237018376 & 55.2214803970355 & 153.191695901127 & -60.0000000000000 & -157.500000000000 \\
\hline
\end{tabular}

sides of equation (11) by $e^{-\xi \wedge_{2}{ }^{\prime} \theta_{2}}$ and equation (18) by $e^{-\xi \wedge_{1} \theta_{1}}$ gives the following relationships:

$e^{-\xi \wedge_{2}{ }^{\prime} \theta_{2}} \mathbf{g}_{S T_{-} 1}(\boldsymbol{\theta})=e^{\xi \wedge_{3}{ }^{\prime} \theta_{3}} e^{\xi \wedge_{4}{ }^{\prime} \theta_{4}} e^{\xi \wedge_{5}{ }^{\prime} \theta_{5}} e^{\xi \wedge_{6}{ }^{\prime} \theta_{6}} e^{\xi \wedge_{7}{ }^{\prime} \theta_{7}}=\mathbf{g}_{S T \_2}(\boldsymbol{\theta})$,

$e^{-\xi \wedge_{1} \theta_{1}} \mathbf{g}_{S T_{-1}}(\boldsymbol{\theta})=e^{\xi \wedge_{3}{ }^{\prime} \theta_{3}} e^{\xi \wedge_{4}{ }^{\prime} \theta_{4}} e^{\xi \wedge_{5}{ }^{\prime} \theta_{5}} e^{\xi \wedge_{6}{ }^{\prime} \theta_{6}} e^{\xi \wedge_{7}{ }^{\prime} \theta_{7}}=\mathbf{g}_{S T_{-} 2}(\boldsymbol{\theta})$.

It can be found that equations (23) and (24) are similar. Hence, the problems of resolving the analytical inverse kinematics of these two 6-DOF manipulators become the problem of resolving the analytical inverse kinematics of a 5-DOF manipulator.

3.3.1. Solving $\theta_{6}$ and $\theta_{7}$. For the sake of analysis, equations (23) and (24) are written in a uniform form:

$$
e^{\xi \wedge_{3}^{\prime} \theta_{3}} e^{\xi \wedge_{4}^{\prime} \theta_{4}} e^{\xi \wedge_{5}^{\prime} \theta_{5}} e^{\xi \wedge_{6}^{\prime} \theta_{6}} e^{\xi \wedge_{7}^{\prime} \theta_{7}}=\mathbf{g}_{S T_{-} 2}(\boldsymbol{\theta}) .
$$

According to the configuration of the SSRMS-type manipulators, the joints in the middle group, joint 6 and joint 7 , are perpendicular to each other. The attitude of the 5-DOF manipulator is decoupled. Hence, two sets of $\theta_{6}, \theta_{7}$, and $\theta_{3}+\theta_{4}+\theta_{5}$ can be obtained by solving kinematics equation (25).

3.3.2. Solving $\theta_{3}, \theta_{4}$, and $\theta_{5}$. Right multiplying both sides of equation (25) by $e^{-\xi \wedge_{7}{ }^{\prime} \theta_{7}} e^{-\xi \wedge_{6}{ }^{\prime} \theta_{6}}$ gives the following relationship:

$$
\mathbf{g}_{S T_{-} 2}(\boldsymbol{\theta}) e^{-\xi \wedge_{7}{ }^{\prime} \theta_{7}} e^{-\xi \wedge_{6}{ }^{\prime} \theta_{6}}=e^{\xi \wedge_{3}{ }^{\prime} \theta_{3}} e^{\xi \wedge_{4}{ }^{\prime} \theta_{4}} e^{\xi \wedge_{5}{ }^{\prime} \theta_{5}}=\mathbf{g}_{S T_{-} 3}(\boldsymbol{\theta}) .
$$

The 5-DOF manipulator degrades to a new 3-DOF planar redundant manipulator. Combining the known $\theta_{3}+$ $\theta_{4}+\theta_{5}$, two sets of $\theta_{3}, \theta_{4}$, and $\theta_{5}$ can be obtained.

Until now, the analytical inverse kinematics of an SSRMS-type manipulator has been solved by using the POE formula and the Paden-Kahan problem. The resolution pro- cess is as follows: resolving the front joints $\longrightarrow$ resolving the end joints $\longrightarrow$ resolving the middle joints. As shown in Figure 9, when locking a front joint (joint 1 or 2) at a fixed position, two sets of solutions for the other (joint 2 or 1) can be solved by using the geometrical relationships and the Paden-Kahan subproblems. Then, for each set of the front joints (joints 1 and 2), two sets of the end joints (joints 6 and 7) and $\theta_{3}+\theta_{4}+\theta_{5}$ can be solved by using algebraic methods. Finally, for every set of the front and end joints (joints 1, 2, 6, and 7), two sets of the middle joints (joints 3, 4 , and 5) can be solved by using algebraic methods. Because of the symmetrical structure of the SSRMS-type manipulators, the solution flow is similar when locking an end joint (joint 6 or 7 ).

\section{Simulation}

To verify the efficiency of the proposed method, simulations including two inverse kinematics cases, two motion planning cases, and an on-orbit kinematics calibration case are carried out on the CMM which will be deployed on the core module of the Chinese space station. The structural parameters of the CMM are set as follows:

$$
\begin{aligned}
& l_{0}=1500 \mathrm{~mm}, \\
& l_{1}=1000 \mathrm{~mm}, \\
& l_{2}=1000 \mathrm{~mm}, \\
& l_{3}=4000 \mathrm{~mm}, \\
& l_{4}=1000 \mathrm{~mm}, \\
& l_{5}=4000 \mathrm{~mm}, \\
& l_{6}=1000 \mathrm{~mm}, \\
& l_{7}=1000 \mathrm{~mm}, \\
& l_{8}=1500 \mathrm{~mm} .
\end{aligned}
$$

4.1. Inverse Kinematics and Motion Planning Cases. According to the configuration analysis in Section 2.3, the analytical inverse kinematics of the CMM can be 


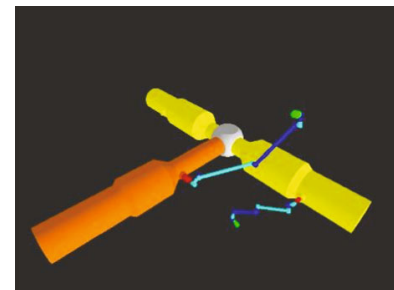

(a) Initial pose

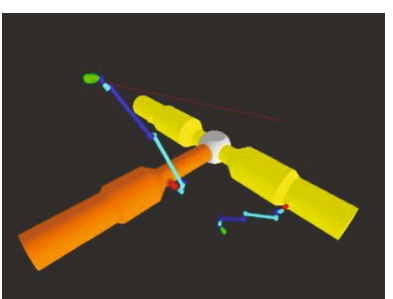

(b) Target pose of the linear trajectory

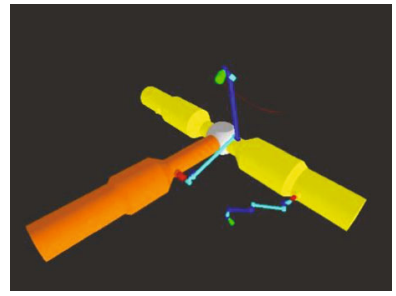

(c) Target pose of the spline curve trajectory

FIGURE 10: Motion planning simulation cases.

obtained only when a front or end joint (joint 1, 2, 6, or 7) is locked. Because of the symmetrical structure of the SSRMS-type manipulators, the cases for locking joints 1 and 2 are taken as examples. First, we arbitrarily select two sets of joint angles within the bounds of the workspace range $(-\pi, \pi)$.

(1) When the first joint is locked at $\theta_{1}^{L}=30^{\circ}$, we set

$$
\begin{aligned}
& \theta_{1}^{L}=30^{\circ}, \\
& \theta_{2}=60^{\circ}, \\
& \theta_{3}=30^{\circ}, \\
& \theta_{4}=50^{\circ}, \\
& \theta_{5}=0^{\circ}, \\
& \theta_{6}=30^{\circ}, \\
& \theta_{7}=22.5^{\circ} .
\end{aligned}
$$

The pose of the tool frame $\mathbf{g}_{S T}(\boldsymbol{\theta})$ in the base frame can be calculated by using the forward kinematics equation (8):

$$
\mathbf{g}_{S T}(\boldsymbol{\theta})=\left[\begin{array}{cccc}
-0.830 & -0.550 & 0.097 & -10694.541 \\
0.123 & -0.350 & -0.929 & 198.467 \\
0.545 & -0.759 & 0.358 & -248.526 \\
0 & 0 & 0 & 1
\end{array}\right]
$$

(2) When the second joint is locked at $\theta_{2}^{L}=45^{\circ}$, we set

$$
\begin{aligned}
& \theta_{1}=30^{\circ}, \\
& \theta_{2}^{L}=45^{\circ}, \\
& \theta_{3}=50^{\circ}, \\
& \theta_{4}=-45^{\circ}, \\
& \theta_{5}=40^{\circ}, \\
& \theta_{6}=60^{\circ}, \\
& \theta_{7}=22.5^{\circ} .
\end{aligned}
$$

The pose of the tool frame $\mathbf{g}_{S T}(\boldsymbol{\theta})$ in the base frame can be calculated by using the forward kinematics equation (8):

$$
\mathbf{g}_{S T}(\boldsymbol{\theta})=\left[\begin{array}{cccc}
-0.520 & -0.636 & -0.570 & -10403.580 \\
0.665 & 0.117 & -0.737 & 5019.108 \\
0.535 & -0.763 & 0.362 & -32.825 \\
0 & 0 & 0 & 1
\end{array}\right] .
$$

Then, taking equations (29) and (31) as known conditions, the analytical inverse kinematics of the CMM can be solved by using the proposed method in Section 3. The corresponding simulation results are listed in Tables 2 and 3, respectively. It can be found that the proposed method can solve all 8 sets of possible solutions when locking joint 1 or 2. Next, we solve the forward kinematics again to calculate the poses of the tool frame corresponding to these 8 sets of 


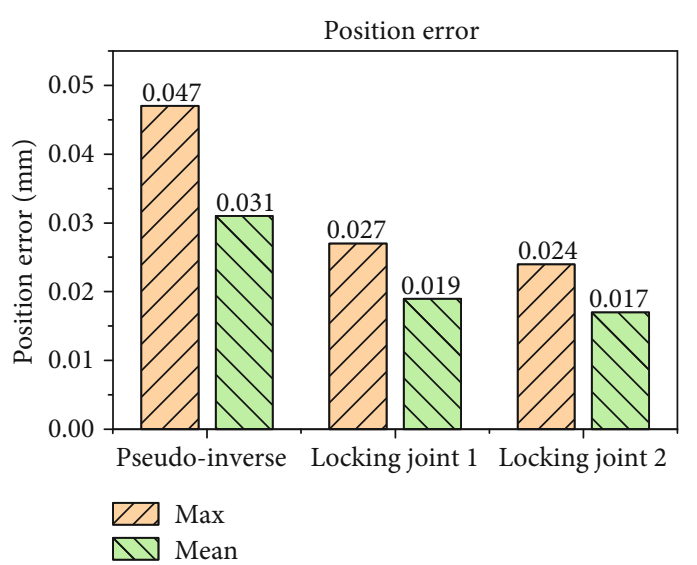

(a) Position error

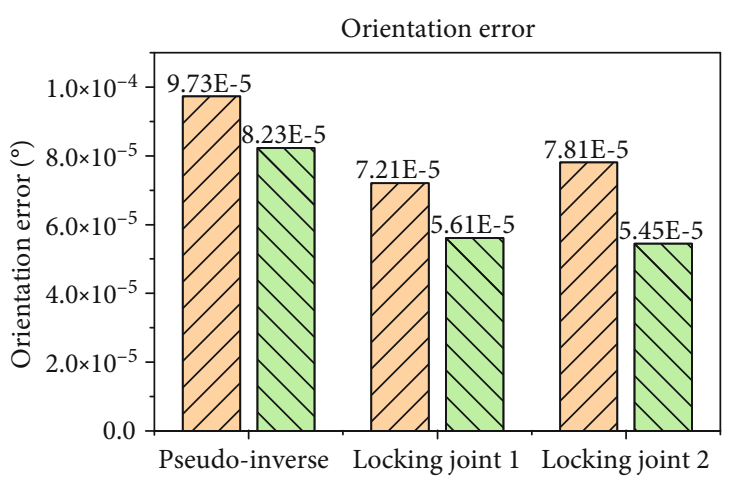

(b) Orientation error

FIGURE 11: The mean position and orientation errors in the motion planning cases.

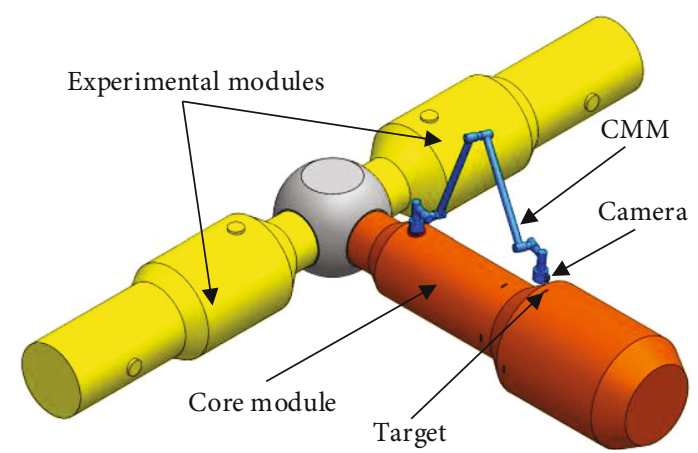

FIGURE 12: Simulation setup of the on-orbit kinematics calibration.

solutions listed in Table 2 or 3 and calculate their corresponding position and orientation errors to evaluate the maximum position and orientation errors. Finally, it can be concluded that when the first joint is locked, the maximum position and orientation errors of the new 6-DOF manipulator are $0.025 \mathrm{~mm}$ and $6.7 \times 10^{-5 \circ}$, and when the second joint is locked, the maximum position and orientation errors of the new 6-DOF manipulator are $0.021 \mathrm{~mm}$ and $6.9 \times 10^{-5 \circ}$.

To further evaluate the computational accuracy of the proposed method, a linear trajectory and a spline curve trajectory for the CMM are employed. As shown in Figure 10, these two trajectories have the same initial pose $\mathbf{T}_{\text {ini }}$, and their target poses are $\mathbf{T}_{\mathrm{tar} \_l i n}$ and $\mathbf{T}_{\mathrm{tar} \_ \text {spl }}$, respectively.

$$
\begin{aligned}
\mathbf{T}_{\mathrm{ini}} & =\left[\begin{array}{llllll}
5852.225157 .88266 .5963 .45113 .55172 .92
\end{array}\right]^{T}, \\
\mathbf{T}_{\text {tar_lin }} & =\left[\begin{array}{llllll}
-2000 & 8000 & 9000 & 0 & 0 & 0
\end{array}\right]^{T}, \\
\mathbf{T}_{\text {tar_spl }} & =\left[\begin{array}{llllll}
3000 & 7500 & 4000 & 0 & 0 & 0
\end{array}\right]^{T} .
\end{aligned}
$$

15001 and 9001 discrete poses on the linear and spline curve trajectories are sampled to calculate the corresponding joint angles by using the proposed method. For comparison, the POE-based pseudo-inverse method is also employed in

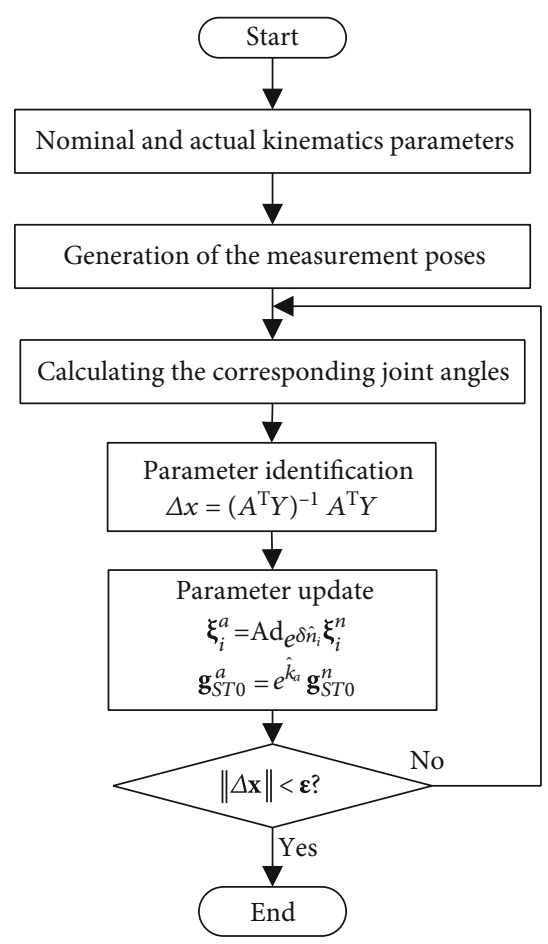

FIGURE 13: Flowchart of the on-orbit kinematics calibration.

the motion planning of these two testing trajectories, and it can be expressed in the following form:

$$
\dot{\boldsymbol{\theta}}=\mathbf{J}^{+} \dot{\mathbf{X}}
$$

where $\dot{\boldsymbol{\theta}}$ denotes the joint velocity column vector, $\mathbf{J}^{+}$is the pseudo-inverse of the Jacobian matrix, and $\dot{\mathbf{X}}$ denotes the velocity column vector of the end effector.

As shown in Figure 11, our proposed method is with higher computational accuracy in solving the inverse kinematics of the CMM than the POE-based pseudo-inverse method. Our proposed method improves the mean position 
TABLE 4: Kinematics parameters of the CMM.

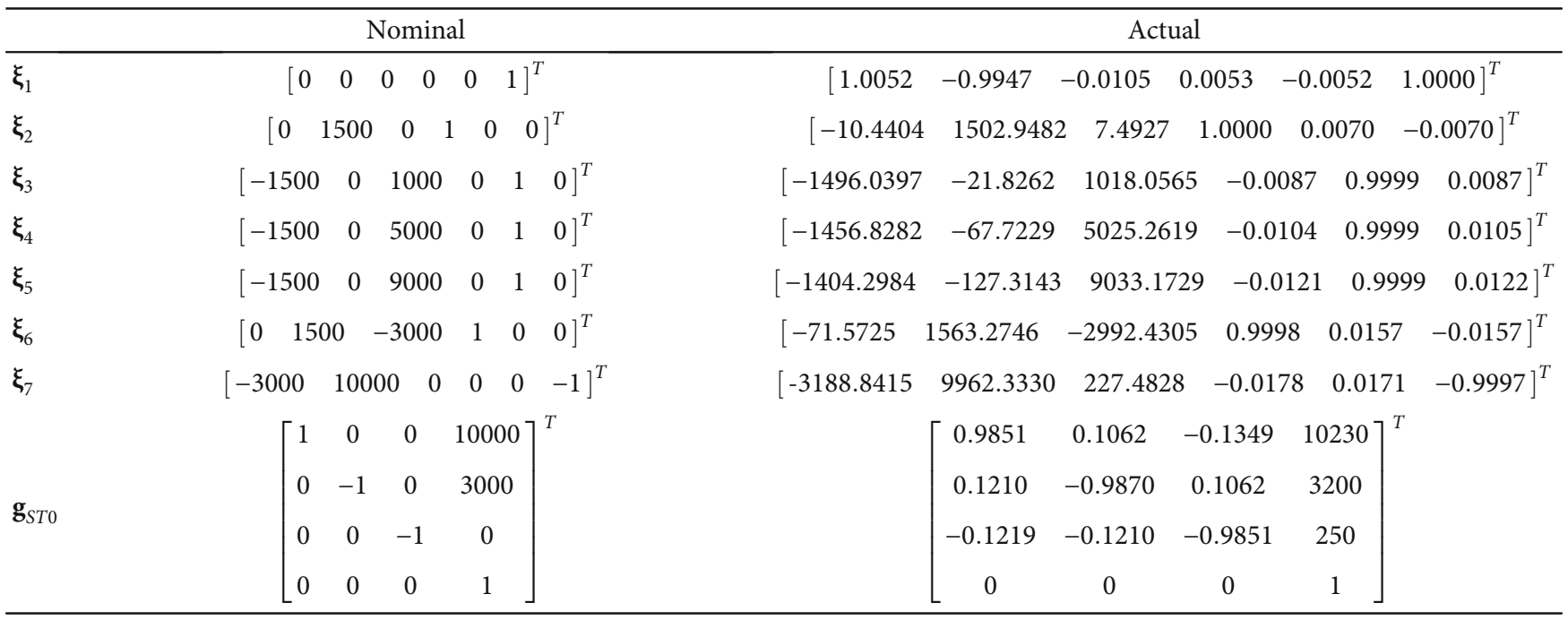

and orientation accuracy by approximately $42 \%$ and $33 \%$, respectively.

Besides, the computational time of the proposed method is also verified. We implement the proposed algorithm to solve the inverse kinematics of the linear trajectory in Figure 10, in VC++ environment (Windows 10 64-bit, Intel Core i5 $9300 \mathrm{H} 2.4 \mathrm{GHz}$ CPU, $8 \mathrm{~GB}$ DDR4 RAM, $500 \mathrm{~GB}$ SSD), and record the computation time of every sampling pose 800 times. When locking the first joint, the mean and maximum computational time of the proposed method is $4.513 \mu \mathrm{s}$ and $5.571 \mu \mathrm{s}$, respectively. When locking the second joint, the mean and maximum computational time of the proposed method is $4.735 \mu \mathrm{s}$ and $5.657 \mu \mathrm{s}$, respectively. The results show that our proposed method has sufficient computational efficiency to solve the analytical solutions of the SSRMS-type manipulators in practical applications.

4.2. On-Orbit Kinematics Calibration Case. SSRMS-type manipulators work in the space environment most of the time. In addition to machining and assembly errors, the huge impact during the launching process and the deformation after long-term use will inevitably cause changes in structural parameters. Therefore, it is essential to conduct on-orbit kinematics calibration for SSRMS-type space manipulators. Due to the singularity of the DH method when two adjacent joints are nearly parallel, the DH method is not suitable for establishing the forward and inverse kinematics of the SSRMS-type manipulators with three adjacent parallel joints.

Although the nonsingularity of the POE formula has been well proven by scholars, to verify that the proposed method based on POE formula has avoided the singularity of the DH method, it is employed in the on-orbit kinematics self-calibration of the CMM. Different from the kinematics calibration of traditional industrial robots, no external measuring equipment is available when carrying out on-orbit kinematics calibration of a space manipulator, the stereo camera mounted on the end effector of an SSRMS-type manipulator is always employed to measure the absolute poses of the end effector [35], as shown in Figure 12. Limited by the measurement range of the stereo camera and the huge structural size of the CMM, the measurement configurations cannot be randomly generated in the joint space but generated in the operational space, and then, the corresponding joint angles for calibration can be calculated by inverse kinematics at every iteration step.

The on-orbit kinematics calibration of the CMM is implemented in MATLAB environment, the simulation flow is shown in Figure 13, the nominal and actual kinematics parameters of the SSRMS-type manipulator are set as Table 4, pose measurements are generated within the measurement range of the cameras mounted on the CMM's end effector, each pose measurement is injected with uniformly distributed noise within $[-2.4,2.4] \mathrm{mm}$ and $[-0.3,0.3]^{\circ}$ to emulate the real situation, and the corresponding joint angles for calibration are calculated using the proposed method instead of the POE-based pseudo-inverse method. In the parameter identification and compensation phases, the adjoint error model [30] is employed to establish the relationship between the kinematics parameter errors $\Delta \mathbf{x}$ and the pose errors of the end effector $\mathbf{y}^{i}$ :

$$
\mathbf{y}^{i}=\mathbf{A}^{i} \Delta \mathbf{x}
$$

where $\mathbf{A}^{i}$ is the Jacobian matrix. Given $m$ end effector pose measurements $\mathbf{y}^{i}$ and the corresponding Jacobian matrix $\mathbf{A}^{i}$, $i=1,2, \cdots, m$, the overall error equation can be expressed as

$$
\mathbf{Y}=\left[\begin{array}{c}
\mathbf{y}^{1} \\
\mathbf{y}^{2} \\
\cdots \\
\mathbf{y}^{m}
\end{array}\right]=\left[\begin{array}{c}
\mathbf{A}^{1} \\
\mathbf{A}^{2} \\
\cdots \\
\mathbf{A}^{m}
\end{array}\right] \Delta \mathbf{x}=\mathbf{A} \Delta \mathbf{x} .
$$




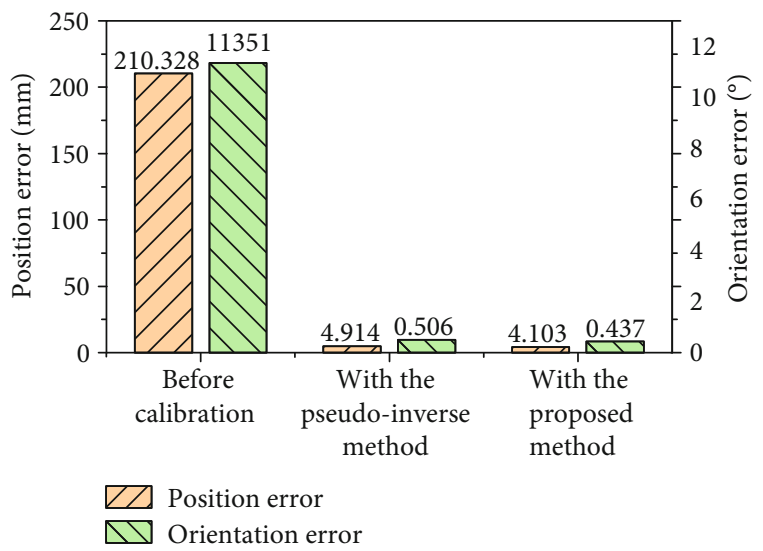

Figure 14: Pose accuracy improvements after calibration.

With enough pose measurements that make A satisfy the full column rank condition, the least square solution of the parameter errors $\Delta \mathbf{x}$ can be solved.

$$
\Delta \mathbf{x}=\left(\mathbf{A}^{T} \mathbf{A}\right)^{-1} \mathbf{A}^{T} \mathbf{Y}
$$

At the end of each iteration step, the kinematics parameters $\xi_{i}^{a}$ and $\mathbf{g}_{S T 0}^{a}$ are updated using the following two equations [30], and they are regarded as the new nominal values in the next iteration step.

$$
\left\{\begin{array}{l}
\xi_{i}^{a}=\operatorname{Ad}_{e^{\delta \eta} \wedge_{i}} \xi_{i}^{n}, \\
\mathbf{g}_{S T 0}^{a}=e^{\mathbf{\eta} \wedge_{s t}} \mathbf{g}_{S T 0}^{n},
\end{array}\right.
$$

where $\boldsymbol{\eta}_{i}$ and $\boldsymbol{\eta}_{s t}$ are variable twists.

The iteration is not terminated until the norm of $\Delta \mathbf{x}$ converges to a preset small threshold $\varepsilon$.

To verify the accuracy of the kinematics calibration, sixty random configurations are employed to calculate the mean position and orientation errors before and after calibration. As shown in Figure 14, compared with the POE-based pseudo-inverse method, the proposed method improves the mean position and attitude accuracy by $9 \%$ and $7 \%$, respectively. Due to the singularity of the $\mathrm{DH}$ method when the adjacent joints are close to parallel, the DH method is not suitable for establishing the actual kinematics of the CMM, and the traditional DH-based calibration algorithm cannot converge to stable values. Therefore, the calibration simulation results show that both the POE-based pseudoinverse method and our proposed method have avoided the singularity of the DH method and can establish the actual kinematics of an SSRMS-type manipulator with 3 adjacent nearly parallel joints, which owes to the smooth mapping between Lie Algebras se(3) and Lie Groups SE(3).

\section{Conclusion}

In this paper, we propose a novel POE-based analytical inverse kinematics method for the SSRMS-type space manipulators. Owing to the smooth mapping between Lie Algebras se(3) and Lie Groups SE(3), the POE formula is employed to solve the analytical inverse kinematics of an SSRMS-type space manipulator to avoid the singular problem of the widely used $\mathrm{DH}$ method. To determine the locked joint (joint $1,2,6$, or 7), the joints of an SSRMS-type manipulator are divided into three categories-the front group, the middle group, and the end group-by configuration analysis. Because of the symmetrical structure, an SSRMS-type space manipulator degrades to two kinds of 6-DOF manipulators when locking a front or end joint. The analytical inverse kinematics of these two 6-DOF manipulators is solved by combining the Paden-Kahan subproblems, geometric methods, and algebraic methods. Finally, to verify the efficiency of the proposed method, it is employed in two analytical inverse kinematics cases, two motion planning cases, and an on-orbit kinematics calibration case. The simulation results show that the proposed method can not only avoid the singularity of the traditional DH method but also are with higher calculation accuracy than the POE-based pseudo-inverse method.

In our future work, we will further examine the singularity configuration when solving the analytical inverse kinematics. Optimizing the motion planning algorithms for the SSRMS-type manipulators by determining the position of the locked joint will also be a research direction.

\section{Data Availability}

The data used to support the findings of this study are included within the article.

\section{Conflicts of Interest}

The authors declare that they have no conflicts of interest.

\section{Acknowledgments}

This work was supported by the National Key R\&D Program of China (2017YFB1300400), the National Natural Science Foundation of China (Grant Nos. 91748201 and 51775011), and the Beijing Natural Science Foundation (3192017).

\section{References}

[1] W. J. Li, D. Y. Cheng, X. G. Liu et al., "On-orbit service (OOS) of spacecraft: a review of engineering developments," Progress in Aerospace Sciences, vol. 108, pp. 32-120, 2019.

[2] A. Flores-Abad, O. Ma, K. Pham, and S. Ulrich, "A review of space robotics technologies for on-orbit servicing," Progress in Aerospace Sciences, vol. 68, pp. 1-26, 2014.

[3] T. Rybus, K. Seweryn, J. Oleś et al., "Application of a planar air-bearing microgravity simulator for demonstration of operations required for an orbital capture with a manipulator," Acta Astronautica, vol. 155, pp. 211-229, 2019.

[4] W. Xu, L. Yan, Z. Hu, and B. Liang, "Area-oriented coordinated trajectory planning of dual-arm space robot for capturing a tumbling target," Chinese Journal of Aeronautics, vol. 32, no. 9, pp. 2151-2163, 2019.

[5] V. Truong, T. Greco, I. Kassam, O. D'Silva, and E. Tan, “A cost effective methodology for building flight spares for robotic life 
extension on the international space station," Acta Astronautica, vol. 162, pp. 405-408, 2019.

[6] P. Colmenarejo, M. Graziano, G. Novelli et al., "On ground validation of debris removal technologies," Acta Astronautica, vol. 158, pp. 206-219, 2019.

[7] G. Chen, Y. Wang, Y. Wang, J. Liang, L. Zhang, and G. Pan, "Detumbling strategy based on friction control of dual-arm space robot for capturing tumbling target," Chinese Journal of Aeronautics, vol. 33, no. 3, pp. 1093-1106, 2020.

[8] Z. NI, J. LIU, Z. WU, and X. SHEN, "Identification of the statespace model and payload mass parameter of a flexible space manipulator using a recursive subspace tracking method," Chinese Journal of Aeronautics, vol. 32, no. 2, pp. 513-530, 2019.

[9] W. F. Xu, Y. She, and Y. S. Xu, "Analytical and semi-analytical inverse kinematics of SSRMS-type manipulators with single joint locked failure," Acta Astronautica, vol. 105, no. 1, pp. 201-217, 2014.

[10] G. Chen, Y. Fu, Q. Jia, B. Yuan, and D. Liu, "A multi-stage regulation strategy of space manipulators with a free-swinging joint failure," Chinese Journal of Aeronautics, vol. 34, no. 5, pp. 573-584, 2021.

[11] J. M. Hollerbach, "Optimum kinematic design for a seven degree of freedom manipulator," International Symposium of Robotics ResearchCambridge, MA: Mit Press, pp. , 1985215222, 1985.

[12] M. Shimizu, H. Kakuya, W. K. Yoon, K. Kitagaki, and K. Kosuge, "Analytical inverse kinematic computation for 7-DOF redundant manipulators with joint limits and its application to redundancy resolution," IEEE Transactions on Robotics, vol. 24, no. 5, pp. 1131-1142, 2008.

[13] H. Liu, "An overview of the space robotics progress in China," Proceedings of the International Symposium on Artificial Intelligence Robotics and Automation in Space, 2014, pp. 15-20, Montreal, Canada, 2014.

[14] B. Siciliano and O. Khatib, Springer Handbook of Robotics, Springer, Berlin, Germany, 2 edition, 2016.

[15] L. G. Huang and Y. T. Li, "Repeatable kinematic control of redundant manipulators: implementation issues," in 8th International Conference on Advanced Robotics. Proceedings. ICAR'97, pp. 147-152, Monterey, CA, USA, 1997.

[16] R. V. Dubey, J. A. Euler, and S. M. Babcock, "Real-time implementation of an optimization scheme for seven-degree-offreedom redundant manipulators," IEEE Transactions on Robotics \& Automation, vol. 7, no. 5, pp. 579-588, 1991.

[17] M. Shah and R. V. Patel, "Inverse Jacobian based hybrid impedance control of redundant manipulators," in International Conference Mechatronics and Automation; 2005, pp. 55-60, Niagara Falls, Ont., Canada, 2005.

[18] Ji Xiang, Congwei Zhong, and Wei Wei, "General-weighted least-norm control for redundant manipulators," IEEE Transactions on Robotics, vol. 26, no. 4, pp. 660-669, 2010.

[19] D. Zu, "Efficient inverse kinematic solution for redundant manipulators," Chinese Journal of Mechanical Engineering, vol. 41, no. 6, pp. 71-75, 2005.

[20] Y. She, W. F. Xu, Z. Y. Wang, and J. Q. Peng, "Inverse kinematics of SSRMS-type manipulators with single joint locked failure," in 2013 IEEE International Conference on Robotics and Biomimetics (ROBIO), pp. 7-12, Shenzhen, China, 2013.
[21] C. Yu, M. Jin, and H. Liu, "An analytical solution for inverse kinematic of 7-DOF redundant manipulators with offsetwrist," in 2012 IEEE International Conference on Mechatronics and Automation, pp. 92-97, Chengdu, China, 2012.

[22] S. Hayati, "Robot arm geometric link parameter estimation," in The 22nd IEEE Conference on Decision and Control, pp. 1477-1483, San Antonio, TX, USA, 1983.

[23] H. W. Stone, Kinematic modeling, identification, and control of robotic manipulators [M.S. thesis], Carnegie Mellon University, New York, USA, 1986.

[24] H. Q. Zhuang and Z. S. Roth, "A linear solution to the kinematic parameter identification of robot manipulators," IEEE Transactions on Robotics and Automation, vol. 9, no. 2, pp. 174-185, 1993.

[25] H. Q. Zhuang, Z. S. Roth, and F. Hamano, "A complete and parametrically continuous kinematic model for robot manipulators," IEEE Transactions on Robotics \& Automation, vol. 8, no. 4, pp. 451-463, 1992.

[26] F. C. Park and K. Okamura, "Kinematic calibration and the product of exponentials formula," in Advances in Robot Kinematics and Computational Geometry, pp. 119-128, Springer Netherlands, Dordrecht, 1994.

[27] K. Okamura and F. C. Park, "Kinematic calibration using the product of exponentials formula," Robotica, vol. 14, no. 4, pp. 415-421, 1996.

[28] I. M. Chen, G. Yang, C. T. Tan, and S. H. Yeo, "Local POE model for robot kinematic calibration," Mechanism and Machine Theory, vol. 36, no. 11-12, pp. 1215-1239, 2001.

[29] G. L. Chen, H. Wang, and Z. Q. Lin, "Determination of the identifiable parameters in robot calibration based on the POE formula," IEEE Transactions on Robotics, vol. 30, no. 5, pp. 1066-1077, 2014.

[30] C. Li, Y. Q. Wu, H. Lowe, and Z. X. Li, "POE-based robot kinematic calibration using axis configuration space and the adjoint error model," IEEE Transactions on Robotics, vol. 32, no. 5, pp. 1264-1279, 2016.

[31] S. Wang, Q. Jia, G. Chen, and D. Liu, "Complete relative pose error model for robot calibration," Industrial Robot: the international journal of robotics research and application, vol. 46, no. 5, pp. 622-630, 2019.

[32] R. Zhao, Z. Shi, Y. Guan, Z. Shao, Q. Zhang, and G. Wang, "Inverse kinematic solution of $6 \mathrm{R}$ robot manipulators based on screw theory and the Paden-Kahan subproblem," International Journal of Advanced Robotic Systems, vol. 15, no. 6, 2018.

[33] Y. C. Wang, Q. Q. Wei, C. W. Hu, and X. L. Ding, “A selfcalibration method for space manipulators based on POE formula," Journal of Beijing University of Aeronautics and Astronautics, vol. 44, no. 11, pp. 2336-2342, 2018.

[34] R. N. Murray, Z. Li, and S. Sastry, A Mathematical Introduction to Robotics Manipulation, CRC Press, Boca Raton, Florida, USA, 1994.

[35] Q. Jia, S. Wang, G. Chen, and H. Sun, "Error modeling in distance and rotation for self-calibration of space robots on orbit," International Journal of Aerospace Engineering, vol. 2019, Article ID 8349048, 16 pages, 2019. 